\title{
Front Matter: Volume 6485
}

, "Front Matter: Volume 6485," Proc. SPIE 6485, Novel In-Plane Semiconductor Lasers VI, 648501 (27 February 2007); doi: 10.1117/12.727505

SPIE Event: Integrated Optoelectronic Devices 2007, 2007, San Jose, California, SPIE. United States 


\title{
PROCEEDINGS OF SPIE
}

\section{Novel In-Plane Semiconductor Lasers VI}

\author{
Carmen Mermelstein \\ David P. Bour \\ Chairs/Editors
}

22-25 January 2007

San Jose, California, USA

Sponsored and Published by

SPIE-The International Society for Optical Engineering

Volume 6485

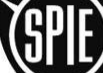

The International Society for Optical Engineering

Proceedings of SPIE-The International Society for Optical Engineering, 9780819465986, v. 6485

SPIE is an international technical society dedicated to advancing engineering and scientific applications of optical, photonic, imaging, electronic, and optoelectronic technologies. 
The papers included in this volume were part of the technical conference cited on the cover and title page. Papers were selected and subject to review by the editors and conference program committee. Some conference presentations may not be available for publication. The papers published in these proceedings reflect the work and thoughts of the authors and are published herein as submitted. The publisher is not responsible for the validity of the information or for any outcomes resulting from reliance thereon.

Please use the following format to cite material from this book:

Author(s), "Title of Paper," in Novel In-Plane Semiconductor Lasers VI, edited by Carmen Mermelstein, David P. Bour, Proceedings of SPIE Vol. 6485 (SPIE, Bellingham, WA, 2007) Article CID Number.

ISSN 0277-786X

ISBN 9780819465986

Published by

SPIE-The International Society for Optical Engineering

P.O. Box 10, Bellingham, Washington 98227-0010 USA

Telephone 1 360/676-3290 (Pacific Time) · Fax 1 360/647-1445

http://www.spie.org

Copyright (C) 2007, The Society of Photo-Optical Instrumentation Engineers

Copying of material in this book for internal or personal use, or for the internal or personal use of specific clients, beyond the fair use provisions granted by the U.S. Copyright Law is authorized by SPIE subject to payment of copying fees. The Transactional Reporting Service base fee for this volume is $\$ 18.00$ per article (or portion thereof), which should be paid directly to the Copyright Clearance Center (CCC), 222 Rosewood Drive, Danvers, MA 01923. Payment may also be made electronically through CCC Online at http://www.copyright.com. Other copying for republication, resale, advertising or promotion, or any form of systematic or multiple reproduction of any material in this book is prohibited except with permission in writing from the publisher. The CCC fee code is 0277$786 \times / 07 / \$ 18.00$.

Printed in the United States of America. 


\section{Contents}

ix Conference Committee

\section{SESSION $1 \quad$ NITRIDE LASERS}

648502 Tunable broad-area InGaN laser diodes in external cavity [6485-01]

K. Komorowska, P. Wisniewski, Institute of High Pressure Physics, Unipress (Poland);

R. Czernecki, TopGaN, Ltd. (Poland); P. Prystawko, Institute of High Pressure Physics, Unipress (Poland); M. Leszczynski, Institute of High Pressure Physics, Unipress (Poland) and TopGaN, Ltd. (Poland); T. Suski, Institute of High Pressure Physics, Unipress (Poland); I. Grzegory, Institute of High Pressure Physics, Unipress (Poland) and TopGaN, Ltd. (Poland); S. Porowski, Institute of High Pressure Physics, Unipress (Poland); S. Grzanka, TopGaN, Ltd. (Poland); M. Maszkowicz, Warsaw Univ. of Technology (Poland); P. Perlin, Institute of High Pressure Physics, Unipress (Poland) and TopGaN, Ltd. (Poland)

648503 Recent progress of high-power GaN-based laser diodes (Invited Paper) [6485-02] T. Kozaki, S. Nagahama, T. Mukai, Nichia Corp. (Japan)

648504 Comprehensive study of reliability of InGaN-based laser diodes [6485-03] L. Marona, M. Sarzynski, P. Wisniewski, High Pressure Research Ctr., Unipress (Poland); M. Leszczynski, High Pressure Research Ctr., Unipress (Poland) and TopGaN, Ltd. (Poland); P. Prystawko, High Pressure Research Ctr., Unipress (Poland); I. Grzegory, High Pressure Research Ctr., Unipress (Poland) and TopGaN, Ltd. (Poland); T. Suski, S. Porowski, High Pressure Research Ctr., Unipress (Poland); R. Czernecki, TopGaN, Ltd. (Poland); G. Kamler, High Pressure Research Ctr., Unipress (Poland); A. Czerwinski, M. Pluska, J. Ratajczak, Institute of Electron Technology (Poland); P. Perlin, High Pressure Research Ctr., Unipress (Poland) and TopGaN, Ltd. (Poland)

648505 High-power operation of inner-stripe GaN-based blue-violet laser diodes (Invited Paper) [6485-04]

M. Ohya, K. Fukuda, I. Masumoto, S. Kohmoto, K. Naniwae, M. Yamada, M. Matsudate, T. Tsukuda, T. Akagawa, C. Sasaoka, NEC Corp. (Japan)

Pagination: Proceedings of SPIE follow an e-First publication model, with papers published first online and then in print and on CD-ROM. Papers are published as they are submitted and meet publication criteria. A unique, consistent, permanent citation identifier (CID) number is assigned to each article at the time of the first publication. Utilization of CIDs allows articles to be fully citable as soon they are published online, and connects the same identifier to all online, print, and electronic versions of the publication.

SPIE uses a six-digit CID article numbering system in which:

- The first four digits correspond to the SPIE volume number.

- The last two digits indicate publication order within the volume using a Base 36 numbering system employing both numerals and letters. These two-number sets start with $00,01,02,03,04,05,06,07,08,09,0 A, 0 B$... OZ, followed by 10-1Z, 20-2Z, etc.

The CID number appears on each page of the manuscript. The complete citation is used on the first page, and an abbreviated version on subsequent pages. 
648506 Investigation and comparison of optical gain spectra of (AI,In)GaN laser diodes emitting in the $375 \mathrm{~nm}$ to $\mathbf{4 7 0} \mathrm{nm}$ spectral range [6485-05]

U. T. Schwarz, H. Braun, Regensburg Univ. (Germany); K. Kojima, M. Funato, Y. Kawakami, Kyoto Univ. (Japan); S. Nagahama, T. Mukai, Nichia Corp. (Japan)

\section{SESSION 2 QUANTUM CASCADE LASERS I}

648507 Quantum-cascade lasers without injector regions (Invited Paper) [6485-06]

A. Friedrich, M.-C. Amann, Technical Univ. of Munich (Germany)

648508 Electronic and thermal properties of Sb-based QCLs operating in the first atmospheric window [6485-07]

M. S. Vitiello, G. Scamarcio, INFM/LIT and Univ. degli Studi di Bari (Italy); V. Spagnolo, INFM/LIT and Politecnico di Bari (Italy); Q. Yang, C. Manz, J. Wagner, Fraunhofer Institute for Applied Solid State Physics (Germany); D. G. Revin, J. Cockburn, Univ. of Sheffield (United Kingdom)

64850A Sub-wavelength antireflection gratings on quantum cascade lasers [6485-09]

A. O. Dirisu, C. F. Gmachl, Princeton Univ. (USA); D. L. Sivco, Lucent Technologies (USA)

\section{SESSION 3 MIDINFRARED AND HIGH-POWER LASERS}

64850B Toward an AIGaAsSb/GalnAsSb/GaSb laser emitting beyond 3 $\mu \mathrm{m}$ [6485-10]

J. Angellier, D. Barat, G. Boissier, F. Chevier, P. Grech, Y. Rouillard, Institut d'Electronique du Sud (France)

64850D Narrow-linewidth high-power Al-free active region $(\lambda=852 \mathrm{~nm})$ DFB laser diodes for atomic clocks and interferometry applications [6485-12]

V. Ligeret, F. J. Vermersch, Alcatel Thales III-V Lab. (France); S. Bansropun, Thales Research and Technology, France (France); M. Lecomte, M. Calligaro, O. Parillaud, M. Krakowski,

Alcatel Thales III-V Lab. (France)

64850E In-phase coherent coupling of tapered lasers in an external Talbot cavity [6485-13] I. Hassiaoui, N. Michel, M. Lecomte, O. Parillaud, M. Calligaro, M. Krakowski, Alcatel Thales III-V Lab. (France)

64850F High-brightness slab-coupled optical waveguide lasers (Invited Paper) [6485-14]

R. K. Huang, J. P. Donnelly, L. J. Missaggia, C. T. Harris, B. Chann, A. K. Goyal,

A. Sanchez-Rubio, T. Y. Fan, G. W. Turner, MIT Lincoln Lab. (USA)

64850 G Very high-power 1310nm InP single-mode distributed feedback laser diode with reduced linewidth [6485-15]

P. Doussiere, C.-L. Shieh, S. DeMars, K. Dzurko, JDS Uniphase Corp. (USA) 
64850J Recombination in quantum dot ensembles (Invited Paper) [6485-18]

P. Blood, H. Pask, I. Sandall, H. Summers, Cardiff Univ. (United Kingdom)

64850K Characteristics of InAs/InGaAsP quantum dot laser diodes lasing at 1.55um [6485-19]

E. Lee, Y. Jang, N. Kim, D. Lee, Chungnam National Univ. (South Korea); S. Pyun, D. Ko,

J. Yoon, W. Jeong, Sungkyunkwan Univ. (South Korea); J. Jang, NanoEpi Technologies

Corp. (South Korea)

64850L Robust passively mode-locked $1.3 \mu \mathrm{m}$ quantum-dot lasers with low timing jitter (Invited Paper) [6485-20]

M. T. Todaro, J.-P. Tourrenc, S. P. Hegarty, C. Kelleher, B. Corbett, G. Huyet, J. G. Mclnerney, National Univ. of Ireland, Cork (Ireland)

SESSION 6 QUANTUM CASCADE LASERS II

$64850 \mathrm{M}$ High-temperature and high-power terahertz quantum-cascade lasers (Invited Paper)

[6485-21]

B. S. Williams, Univ. of California, Los Angeles (USA); Q. Qin, S. Kumar, Q. Hu, Massachusetts Institute of Technology (USA); J. L. Reno, Sandia National Labs. (USA)

64850N MOCVD growth and regrowth of quantum cascade lasers (Invited Paper) [6485-22]

F.-S. Choa, L. Cheng, X. Ji, Univ. of Maryland, Baltimore County (USA); Z. Liu, D. Wasserman,

S. S. Howard, C. F. Gmachl, Princeton Univ. (USA); X. Wang, J. Fan, AdTech Optics (USA);

J. Khurgin, The Johns Hopkins Univ. (USA)

64850P High-performance continuous wave quantum cascade lasers with widely spaced operation frequencies [6485-24]

A. Wittmann, Univ. de Neuchâtel (Switzerland); L. Hvozdara, S. Blaser, Alpes Lasers SA

(Switzerland); M. Giovannini, J. Faist, D. Hofstetter, M. Beck, Univ. de Neuchâtel

(Switzerland); E. Gini, ETH Zürich (Switzerland)

\section{SESSION 7 NOVEL DEVICES}

64850Q Nonselective oxidation of GaAs-based III-V compound semiconductor heterostructures for in-plane semiconductor lasers (Invited Paper) [6485-25]

D. Liang, J. Wang, D. C. Hall, Univ. of Notre Dame (USA)

$64850 S$ Properties of GalnNAsSb narrow ridge waveguide laser diodes in continuous-wave operation at 1.55um (Invited Paper) [6485-27]

J. A. Gupta, P. J. Barrios, G. Pakulski, G. C. Aers, J. A. Caballero, D. Poitras, X. Wu, National Research Council of Canada (Canada)

$64850 U$ New THz sources for bio-medical imaging (Invited Paper) [6485-29]

J. S. Harris, A. GU, S. M. Kim, Stanford Univ. (USA) 
64850V Nonlinear optics with intersubband transitions in high-band offset heterostructures (Invited Paper) [6485-30]

F. Xie, V. R. Chaganti, A. Belyanin, Texas A\&M Univ. (USA); A. Idziaszek, J. Kono, Rice Univ. (USA); S. Sasa, M. Inove, Osaka Institute of Technology (Japan)

SESSION 9 NOVEL DEVICES AND PHYSICS

64850X High-frequency nanophotonic devices (Invited Paper) [6485-32]

D. Bimberg, G. Fiol, C. Mever, M. Laemmlin, M. Kuntz, Technische Univ. Berlin (Germany)

$64850 Z$ High power pure-blue semiconductor lasers (Invited Paper) [6485-45]

O. Goto, Sony Shiroishi Semiconductor, Inc. (Japan); S. Tomiya, Sony Corp. (Japan);

Y. Hoshina, T. Tanaka, M. Ohta, Y. Ohizumi, Y. Yabuki, Sony Shiroishi Semiconductor, Inc.

(Japan); K. Funato, M. Ikeda, Sony Corp. (Japan)

\section{SESSION 10 SILICON OPTOELECTRONICS I: JOINT SESSION WITH CONFERENCE 6477}

$648510 \mathrm{Si} / \mathrm{Ge}$ platform for lasers, amplifiers, and nonlinear optical devices based on the Raman effect (Invited Paper) [6485-35]

R. Claps, Neptec Optical Solutions (USA); D. Dimitropoulos, V. Raghunathan, S. Fathpour, B. Jalali, Univ. of California, Los Angeles (USA); B. Jusserand, Univ. Pierre et Marie Curie (France)

648511 Monolithic integrated ring resonator Raman silicon laser and amplifier (Invited Paper) [6485-36]

H. Rong, S. XU, Y.-H. Kuo, V. Sih, Intel Corp. (USA); O. Cohen, O. Raday, Intel Corp. (Israel); M. Paniccia, Intel Corp. (USA)

648513 Laser charactersitics and gain properties of the novel Ga(NAsP)/GaP material system for the integration to Si (Invited Paper) [6485-38]

B. Kunert, K. Volz, W. Stolz, Philipps Univ. (Germany)

SESSION 11 SILICON OPTOELECTRONICS II: JOINT SESSION WITH CONFERENCE 6477

648514 Quantum dot lasers and integrated guided wave devices on Si (Invited Paper) [6485-39]

J. Yang, Z. Mi, P. Bhattacharya, Univ. of Michigan (USA)

648515 High-temperature silicon evanescent lasers (Invited Paper) [6485-40]

J. E. Bowers, H. Park, A. W. Fang, Univ. of California, Santa Barbara (USA); R. Jones, Intel

Corp. (USA); O. Cohen, Intel Corp. (Israel); M. J. Paniccia, Intel Corp. (USA)

\section{SESSION $12 \quad$ NEAR IR AND RED LASERS}

$648516 \quad 670 \mathrm{~nm}$ semiconductor lasers for lithium spectroscopy with 1W [6485-41]

R. Häring, Toptica Photonics AG (Germany); B. Sumpf, G. Erbert, G. Tränkle, FerdinandBraun-Institut für Höchstfrequenztechnik (Germany); F. Lison, W. G. Kaenders, Toptica

Photonics AG (Germany) 
$648517670 \mathrm{~nm}$ tapered lasers and amplifiers with output powers $\mathrm{P} \geq 1 \mathrm{~W}$ and nearly diffractionlimited beam quality [6485-42]

B. Sumpf, G. Erbert, J. Fricke, P. Froese, Ferdinand-Braun-Institut für Höchstfrequenztechnik (Germany); R. Häring, W. G. Kaenders, Toptica Photonics AG (Germany); A. Klehr, Ferdinand-Braun-Institut für Höchstfrequenztechnik (Germany); F. Lison, Toptica Photonics AG (Germany); P. Ressel, H. Wenzel, M. Weyers, M. Zorn, G. Tränkle, Ferdinand-Braun-Institut für Höchstfrequenztechnik (Germany)

648518 Highly strained InGaAs lasers grown by MOVPE with low threshold current density [6485-43] W. C. Chen, Y. K. Su, R. W. Chuang, M. C. Tsai, National Cheng Kung Univ. (Taiwan)

648519 Lifetime prediction of diode lasers with different aging behavior [6485-44] Y. Li, Consultant (USA)

Author Index 
Downloaded From: https://www.spiedigitallibrary.org/conference-proceedings-of-spie on 26 Apr 2023

Terms of Use: https://www.spiedigitallibrary.org/terms-of-use 


\title{
Conference Committee
}

\author{
Symposium Chair \\ Yakov Sidorin, Photineer Technology Group (USA) \\ Symposium Cochair
}

Ali Adibi, Georgia Institute of Technology (USA)

Program Track Chair

Daniel K. Johnstone, Virginia Commonwealth University (USA)

Conference Chairs

Carmen Mermelstein, Reute (Germany)

David P. Bour, BridgeLux Corporation (USA)

Program Committee

Yasuhiko Arakawa, The University of Tokyo (Japan)

Dan Botez, University of Wisconsin/Madison (USA)

Federico Capasso, Harvard University (USA)

Gary A. Evans, Southern Methodist University (USA)

Claire F. Gmachl, Princeton University (USA)

Michael Kneissl, Technische Universität Berlin (Germany)

Hui C. Liu, National Research Council Canada (Canada)

Luke J. Mawst, University of Wisconsin/Madison (USA)

Jerry R. Meyer, Naval Research Laboratory (USA)

Mario J. Paniccia, Intel Corporation (USA)

Peter M. Smowton, Cardiff University (United Kingdom)

\section{Session Chairs}

$1 \quad$ Nitride Lasers

David P. Bour, BridgeLux Corporation (USA)

2 Quantum Cascade Lasers I

Federico Capasso, Harvard University (USA)

3 Midinfrared and High-Power Lasers

Carmen Mermelstein, Reute (Germany)

$4 \quad$ High-Power Lasers

Manoj Kanskar, Alfalight, Inc. (USA) 
Quantum Dots

Peter M. Smowton, Cardiff University (United Kingdom)

6 Quantum Cascade Lasers II

Jérôme Faist, Université de Neuchâtel (Switzerland)

$7 \quad$ Novel Devices

Jerry R. Meyer, Naval Research Laboratory (USA)

8 Quantum Cascade Lasers III

Yasuhiko Arakawa, The University of Tokyo (Japan)

9 Novel Devices and Physics

Kenneth B. Crozier, Stanford University (USA)

10 Silicon Optoelectronics I: Joint Session with Conference 6477

Mario J. Paniccia, Intel Corp. (USA)

11 Silicon Optoelectronics II: Joint Session with Conference 6477

Bahram Jalali, University of California, Los Angeles (USA)

12 Near-IR and Red Lasers

Michael Kneissl, Technische Universität Berlin (Germany) 